\title{
EFEITO DA ADIÇÃO DE FIBRAS DE AÇO E POLIMÉRICAS NA RESISTÊNCIA À COMPRESSÃO DO CONCRETO DE ULTRA ALTO DESEMPENHO
}

\author{
Effect of steel and polymeric fibers addition in compressive strength of ultra high \\ performance concrete
}

\author{
Carlos Eduardo Tino Balestra ${ }^{1}$, Jennifer Stephane Ozelame ${ }^{2}$, Gustavo Savaris ${ }^{3}$
}

Recebido em 12 de março de 2019; aceito em 08 de agosto de 2019; disponível on-line em 11 de maio de 2020.

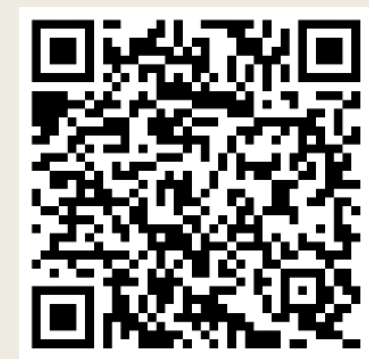

PALAVRAS CHAVE:

Fibras;

Resistência à compressão; Deformação residual; Ultra alto desempenho;

Concreto.

\section{KEYWORDS:}

Fibers;

Compressive strength; Residual strain; Ultra high performance; Concrete.

\section{* Contato com o autor:}

${ }^{1}$ e-mail: carlosbalestra@utfpr.edu.br (C. E. T. Balestra)

Engenheiro Civil, Doutor em Infraestrutura Aeronáutica, Professor Adjunto, Universidade Tec. Federal do Paraná - Câmpus Toledo

${ }^{2}$ e-mail: jennifer.ozelame@hotmail.com (J. S. Ozelame)

Graduanda em Engenharia Civil, Universidade Tecnológica Federal do Paraná - Câmpus Toledo.

${ }^{3}$ e-mail: gsavaris@utfpr.edu.br (G. Savaris)

Engenheiro Civil, Doutor em Engenharia Civil, Professor Adjunto, Universidade Tecnológica Federal do Paraná - Câmpus Toledo.
ABSTRACT: The demand for materials with higher performance than those usually used in civil construction stimulate researches aimed at the development of new technologies. In this context, ultra high performance fibers reinforced concrete (UHPFRC) stands out due to the high compressive and tensile strengths and higher ductility compared to concrete. The present work had as main objective to evaluate experimentally the compressive strength and post - rupture behavior of UHPFRC with of these material in the current constructive processes. UHPFRC mixtures with steel fibers and polyethylene fibers using $0.5 \%$ and $1 \%$ fiber volumes were defined from an ultra high performance concrete mixture. The results obtained were compared to a reference concrete without fibers, demonstrating that the fibers improve the ductility of the concrete and provide a residual resistance after the exemplar fracture, recovery peaks due to the better adhesion of the fibers to the concrete. In addition, for both fibers, a greater amount of fibers helped to avoid sudden ruptures observed after concrete without fibers reached their resistant capacity. different types and dosages of fibers, using materials available in Brazil, aiming the use 


\section{INTRODUÇÃO}

A constante busca por materiais de alto desempenho é a força motriz por trás do desenvolvimento de novos materiais ( $\mathrm{Yl}$ et al., 2012). Neste ponto, o concreto apresenta-se como um dos principais materiais utilizados em sistemas estruturais na construção civil, graças não apenas às suas propriedades mecânicas, mas também devido a outros aspectos que tangem vertentes econômicas, de segurança e de durabilidade (MEHTA; MONTEIRO, 2006; NEVILLE, 2010). Todavia, as estruturas em concreto apresentam limitações relacionadas ao uso convencional do mesmo, dada sua baixa resistência à tração e quase nenhuma ductilidade. Neste sentido, os Concretos de Pós Reativos (CPR), pertencentes à família de concretos de ultra alto desempenho (UHPC) se apresentam como um material capaz de transpor estas limitações apresentadas pelo concreto convencional (YANG, JOH E KIM, 2010).

Os concretos de ultra alto desempenho (UHPC) apresentam-se como um dos compósitos cimentícios mais avançados para fins estruturais no âmbito da engenharia civil, sendo resultado de pesquisas iniciadas na década de 90 que visam concretos de elevado desempenho, principalmente no que tange sua resistência e durabilidade. (RICHARD; CHEYREZY, 1995; YI et al. 2012, ZBED, 2013).

Os UHPC são caraterizados em primeira instância pela granulometria dos materiais que os constituem, compostos por cimento Portland, sílica ativa, areia de quartzo (com dimensão máxima limitada a $600 \mu \mathrm{m}$ ) e pó de quartzo, além do uso de aditivo superplastificante e baixa relação água/cimento, da ordem de 0,2. Desta forma, obtêm-se resistências à compressão da ordem de $150 \mathrm{MPa}$ (WILLE et al., 2011), resultando em resistências à compressão e à tração até 16 e 7 vezes superior às resistências apresentadas por um concreto convencional, respectivamente (WANG et al. 2015).

Para atingir elevados patamares de resistência, busca-se no UHPC um material com o mínimo de defeitos possível como, por exemplo, poros e fissuras. Assim, aspectos como: a minimização da porosidade, obtida através da otimização da mistura granular visando um maior empacotamento de partículas; a densificação da matriz cimentícia; por conta dos produtos de hidratação formados graças à intensa atividade pozolânica dos constituintes; e a adoção de procedimentos de cura térmica e aplicação de pressão no concreto em estado fresco, torna possível a obtenção de elevadas resistências por parte dos UHPC (ZBED, 2013; WANG et al., 2015). Sob esta perspectiva, a adoção de um tratamento térmico durante o processo de cura cria um ambiente favorável à potencialização das reações pozolânicas entre a sílica ativa e o hidróxido de cálcio, onde, neste caso, a sílica ativa consome os cristais de hidróxido de cálcio resultando na formação de silicato de cálcio hidratado, contribuindo assim para as elevadas resistências observadas no UHPC em comparação a um concreto convencional. Habel et al. (2006) pontuam que as diferenças existentes entre o UHPC e concretos convencionais advêm de significativas alterações na cinética das reações de pozolânicas e, concomitantemente a isso, em mudanças latentes nas propriedades mecânicas, uma vez que o desenvolvimento das propriedades mecânicas do UHPC atinge seu o estágio final em praticamente 90 dias, graças ao rápido desenvolvimento do grau das reações pozolânicas, levando a maiores resistências em um menor espaço de tempo.

Além disso, a ausência de agregados graúdos, com diâmetros da ordem de $4 \mathrm{~mm}$ ou superiores, reduz a espessura da zona de transição na interface dos agregados com a pasta de cimento, assim, a microfissuração presente nesta zona em um concreto convencional não é observada no UHPC, o que contribui para aumentar sua resistência em relação a um concreto convencional. (WILLE et al, 2011; ZBED, 2013; AZAD et al, 2013).

Recentemente, pesquisas tem avaliado o UHPC quanto à influência de diferentes tipos e dosagens de cimentos (DILS et al., 2013; ALKAYSE et al., 2016; WANG et al., 2017), adições minerais (RONG et al., 2014; SOLIMAN \& TAGNIT-HAMOU, 2017) e superplastificantes (SCHRÖFL et al., 2012), 
além de estudos sobre o melhor arranjo físico das partículas dos materiais que compõem o UHPC, definidos como empacotamento de partículas (LARRARD \& SEDRAN, 1994; BONNEAU et al., 2000; KOUTNÝ et al., 2016), uso de fibras (SU et al., 2016; ZHOU \& UCHIDA, 2017), influência do preparo e cura na resistência do UHPC s CPR's (KODUR et al., 2016; MOSTOFINEJAD et al., 2016), características do UHPC em estado fresco ( $\mathrm{CHOI}$ et al., 2016) e aspectos de durabilidade (WANG et al., 2012; TAFRAOUI et al., 2016).

Entre estas pesquisas deve ser dado destaque àquelas relacionadas à adição de fibras, de diferentes materiais e morfologias, destinadas a melhorar as propriedades mecânicas do UHPC, principalmente no que tange sua resistência após o inicio da fissuração. Máca et al. (2013) apresentam uma investigação experimental acerca das propriedades de concretos de ultra alto desempenho reforçados com fibras (UHPFRC), onde o emprego de fibras metálicas resultou em melhoria das propriedades mecânicas de um UHPFRC em relação à um concreto convencional. Independentemente do tipo e dosagem de fibra utilizada é consensual entre os estudos apresentados na literatura que sua aplicação leva a melhoras significativas nas propriedades mecânicas de concretos de ultra alto desempenho (YANG et al., 2010; HASSAN et al., 2012; WILLE; NAAMAN, 2011; AZAD; HAKEEM, 2013; KUSUMAWARDANINGSIH et al., 2015). Os efeitos de ancoragem das fibras no concreto são fundamentais para melhorar as propriedades mecânicas do mesmo, onde se tem observado que fibras de maior comprimento acabam levando a uma maior ancoragem junto ao concreto levando a benefícios nas propriedades mecânicas do concreto antes e após o surgimento de fissuras (YOO; BANTHIA, 2016).

Yang, Joh \& Kim (2010) avaliaram a utilização de fibras de aço retas e o método de lançamento do UHPFRC nas fôrmas no comportamento de vigas submetidas a um ensaio de flexão. Os resultados demonstraram que as vigas cujo UHPFRC foi colocado a partir de suas extremidades apresentaram maior capacidade portante, e consequentemente melhor desempenho estrutural, comparada as vigas cujo UHPFRC foi colocado na fôrma a partir de seu centro, uma vez que o fluxo de concreto na fôrma acaba por definir a orientação das fibras em seu interior. Este efeito foi comprovado por Zhou e Uchida (2017) através de tomografias, onde o lançamento do concreto a partir da extremidade dos moldes de vigas fez com que as fibras de orientassem paralelamente à superfície inferior das formas, entretanto, à medida que a fôrma foi preenchida, o fluxo ascendente do concreto fez com que as fibras se orientassem de forma obliqua à superfície inferior da forma, levando a concluir assim que a orientação das fibras é intimamente relacionada ao fluxo percorrido pelo concreto. Hassan, Jones \& Mahmud (2012) e Kusumawardaningsih et al. (2015) realizaram ensaios para determinação da resistência à tração direta de corpos de prova dotados de UHPFRC com um volume de $2 \%$ de fibras de aço, constatando a melhora da resistência à tração do concreto de forma significativa e ainda auxílio no controle de abertura de fissuras após esgotar sua capacidade resistente.

Xia et al. (2011) apresentam um estudo referente à análise de falhas de vigas constituídas por UHPFRC devido ao cisalhamento. Foram executados ensaios de aderência entre uma barra e o UHPFRC e ensaios em sistemas de tabuleiros para pontes reforçados com longarinas e transversinas constituídas com o UHPFRC com armaduras longitudinais sob diferentes tipos de ancoragem. Os autores observaram que o modo de fratura por cisalhamento foi dominante nas vigas e sistemas ensaiados, entretanto a fratura não ocorreu de forma abrupta graças à presença das fibras. Ainda sob esta perspectiva da análise de sistemas de tabuleiros de pontes com longarinas e transversinas, podemos citar os estudos de Saleem et al. (2012) que realizaram um estudo a respeito do comportamento de tais sistemas estruturais com a utilização de diferentes geometrias. Analogamente a Xia et al. (2011) os autores concluem que o modo de fratura foi governado pelo cisalhamento mas, não de forma abrupta.

Dentre a grande variedade de estudos 
referentes aos efeitos das fibras apresentados na literatura, a maior parcela dos estudos trata da aplicação de fibras metálicas, sendo uma parcela menor os estudos que tratam da aplicação de fibras com a mesma geometria, porém de diferentes materiais. Yang et al. (2008) apresenta-se como um dos trabalhos que utilizou fibras metálicas e poliméricas para a verificação das propriedades mecânicas de UHPFRC, observando que o uso de fibras de aço melhoraram significativamente as propriedades mecânicas do UHPC, ao contrário das fibras de polipropileno Desta forma, o presente trabalho tem como objetivo analisar não apenas a melhora das propriedades mecânicas proporcionadas por fibras de aço e poliméricas ao CPR até o esgotamento de sua capacidade última, porém avaliar também a forma de ruptura de corpos de prova e a resistência residual após o concreto atingir sua capacidade máxima à compressão.

\section{PROGRAMA EXPERIMENTAL}

\subsection{MATERIAL E PROPORÇÃO DA MISTURA}

Neste estudo, materiais comercializados no mercado brasileiro foram empregados para produção de UHPFRC, sendo constituído por cimento Portland tipo V-ARI (equivalente ao ASTM Tipo III), areia de quartzo, sílica ativa, pó de quartzo e aditivo superplastificante a base de éter policarboxilato. A dimensão máxima característica e as características químicas, determinadas por espectroscopia de fluorescência de raios- $X$, da areia de quartzo, da sílica ativa e do pó de quartzo são apresentadas na Tabela 1.

A Tabela 2 apresenta a proporção de materiais da mistura, definida a partir de um estudo conduzido para determinar a relação proporcional entre os constituintes do UHPC, apresentado em Ozelame (2017). Dois tipos de fibras retas e lisas foram utilizados para produção do UHPFC, sendo fibras retas de aço com $13 \mathrm{~mm}$ de comprimento e fibras de polietileno de mesmo comprimento (Figura 1).

Para produção de UHPFC os materiais secos foram inicialmente misturados por 5 minutos, em um misturador planetário, sendo posteriormente realizada a adição de água e superplastificante, onde a mistura se processou por mais 10 minutos. Posterior à mistura dos materiais, diferentes dosagens de fibras foram adicionadas ao concreto, procedendo a mistura por mais 5 minutos de modo que as fibras fossem completamente dispersas no concreto.

TABELA 1: Composição química da areia de quartzo, sílica ativa e pó de quartzo.

\begin{tabular}{ccccccc} 
Constituinte & $\begin{array}{c}\text { Dimensão máxima característica } \\
(\mu \mathrm{m})\end{array}$ & $\begin{array}{c}\mathrm{SiO}_{2} \\
(\%)\end{array}$ & $\begin{array}{c}\mathrm{Fe}_{2} \mathrm{O}_{3} \\
(\%)\end{array}$ & $\begin{array}{c}\mathrm{Al}_{2} \mathrm{O}_{3} \\
(\%)\end{array}$ & $\begin{array}{c}\mathrm{TiO}_{2} \\
(\%)\end{array}$ & $\begin{array}{c}\text { Perda ao } \\
\text { fogo (\%) }\end{array}$ \\
\hline Areia de quartzo & 600 & 99,62 & - & - & - & 0,07 \\
\hline Sílica ativa & 45 & 99,68 & 0,045 & 0,230 & 0,028 & 0,11 \\
\hline Pó de quartzo & 25 & 99,64 & - & - & - & 0,08 \\
\hline
\end{tabular}

TABELA 2: Proporção de materiais para UHPC, em massa.

\begin{tabular}{cccccc} 
Cimento & Sílica ativa & Areia de quartzo & Pó de quartzo & $\begin{array}{c}\text { Superplastificant } \\
\text { e }\end{array}$ & Água \\
\hline 1 & 0,26 & 1,11 & 0,28 & 0,03 & 0,26
\end{tabular}



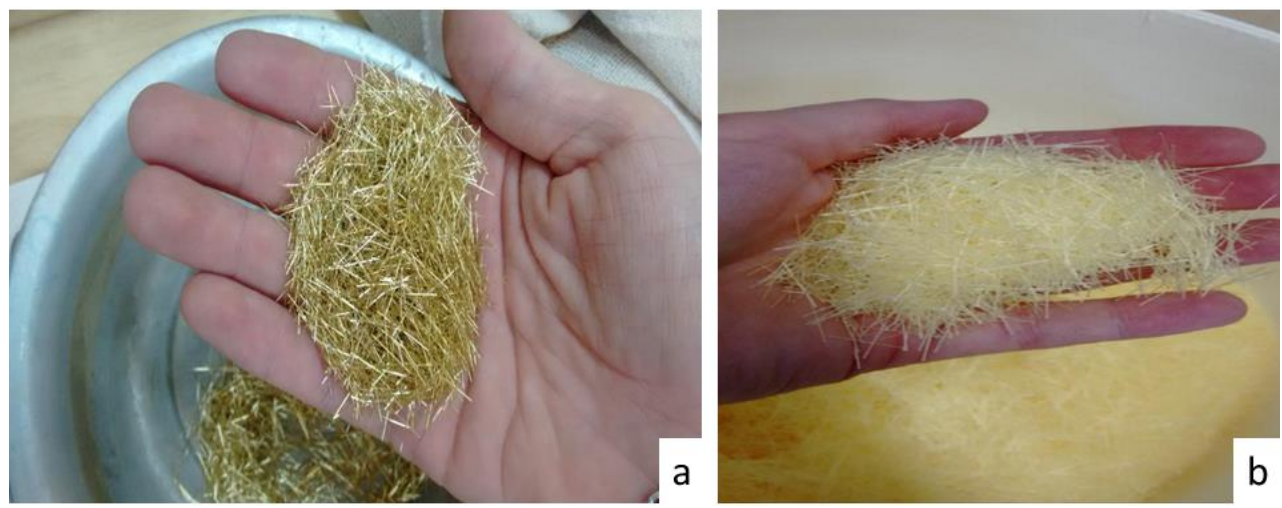

FIGURA 1: Fibras adicionadas ao concreto (a) Fibras de aço (b) Fibras de polietileno. FONTE: Autoria própria.

Os concretos foram classificados em grupos de acordo com a porcentagem de fibras em relação ao volume adicionado, conforme nomenclatura apresentada na Tabela 3, segundo sua respectiva dosagem e tipo de fibra utilizada, onde, as letras representam o tipo de fibra utilizado (NF - sem fibras; SF - Fibras de aço; PF - fibras de polietileno) e os números indicam a percentagem de fibra utilizada ( $0 \%$ - 0, 0,5\% - 05 e 1\% - 1), assim, por exemplo, o grupo identificado como SF1 corresponde ao concreto com $1 \%$ de fibras de aço retas. Logo após a produção dos concretos foram moldados 6 corpos de prova cilíndricos $5 \times 10 \mathrm{~cm}$ (diâmetro x altura) para cada grupo (Figura 2), sendo estes corpos de prova encaminhados à condição de cura à vapor a uma temperatura de $90{ }^{\circ} \mathrm{C}$ por $72 \mathrm{~h}$ e, posteriormente, para cura em câmara úmida, a uma temperatura de $23+2 \circ \mathrm{C}$ e umidade relativa mínima de $95 \%$, até a idade de 28 dias quando os ensaios de compressão foram realizados para avaliação de suas propriedades mecânicas. O procedimento de cura a vapor foi escolhido tendo em vista potencializar a atividade pozolânica da sílica ativa conforme descrito na literatura (Richard \& Cheyrezi, 1995).

\section{TABELA 3: Identificação dos grupos de corpos de prova.}

\begin{tabular}{cl}
\hline Grupo & Descrição \\
\hline NF & Corpos de prova de condição de referência sem fibras \\
\hline SF05 & Corpos de prova com adição de $0,5 \%$ fibras de aço \\
\hline SF1 & Corpos de prova com adição de $1 \%$ fibras de aço \\
\hline PF05 & Corpos de prova com adição de $0,5 \%$ fibras de polietileno \\
\hline PF1 & Corpos de prova com adição de $0,5 \%$ fibras de polietileno \\
\hline
\end{tabular}

FONTE: Autoria própria.

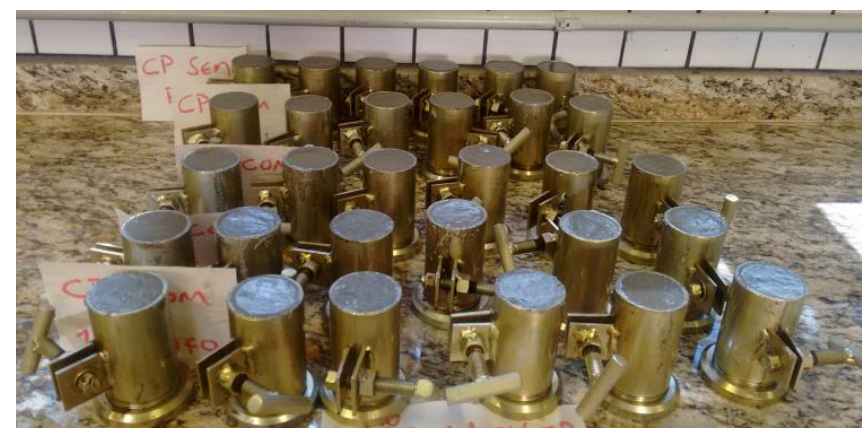

FIGURA 2: Corpos de prova moldados com diferentes dosagens de fibras de aço e polietileno. FONTE: Autoria própria. 


\subsection{RESISTÊNCIA À COMPRESSÃO AXIAL}

Ao atingir 28 dias de idade, os corpos de prova foram retirados da câmara de cura e submetidos ao ensaio de compressão axial em uma máquina universal de ensaios. Durante este ensaio a carga aplicada e a deformação axial foram instrumentadas, possibilitando determinar a resistência máxima do concreto e a resistência residual após o concreto atingir sua capacidade máxima.

\section{RESULTADOS E DISCUSSÃO}

Para avaliar a confiabilidade dos resultados e determinar se as diferenças encontradas entre as diferentes misturas são estatisticamente significativas, foi realizada uma análise de variância, ANOVA, com um nível de confiança de $95 \%$.

\subsection{RESISTÊNCIA À COMPRESSÃO AXIAL}

Na Figura 3 são apresentadas as formas de ruptura dos corpos de prova, sendo na Figura $3 a$ a ruptura típica do grupo de concreto NF, na Figura $3 \mathrm{~b}$ a ruptura típica de concretos pertencentes ao grupo SF e PF com 0,5\% de fibras e na Figura $3 c$ a ruptura típica do grupo de concretos SF e PF com $1 \%$ de fibras.

Inicialmente pode-se observar, através de uma análise da Figura 3, que os corpos de prova que não continham fibras apresentaram uma ruptura brusca ao atingem sua máxima resistência no ensaio de compressão, com planos de ruptura bem delimitados e destacamento completo entre as partes dos corpos de prova (Figura 3a). Os corpos de prova com $0,5 \%$ de fibras em seu volume, independentemente desta ser polimérica ou de aço, apresentaram ruptura brusca, porém sem que fossem constatadas faces planas de ruptura e destacamentos completos (Figura 3b). Tal fato decorre dos efeitos de aderência das fibras ao concreto dos corpos de prova, que permitiram que o mesmo não apresentasse destacamento entre as camadas dos corpos de prova. Tal comportamento demonstra-se importante do ponto de vista estrutural, uma vez que, na eminência da ruína, a estrutura contendo fibras apresentará um nível de ductilidade e fissuração superior à estrutura isenta de fibras. Em corpos de prova com $1 \%$ de fibras este efeito foi ainda mais evidente, onde apenas fissuras são observadas nos corpos de prova, sem que haja um destacamento das partes do mesmo (Figura 3c). Assim, considerando que os concretos de ultra alto desempenho apresentam ruptura brusca, estes resultados demonstram que 0 aumento na quantidade de fibras é benéfico em termos de segurança estrutural, pois as estruturas produzidas com concreto reforçado com fibras acabam apresentando melhor desempenho em relação à estruturas de concreto sem fibras.

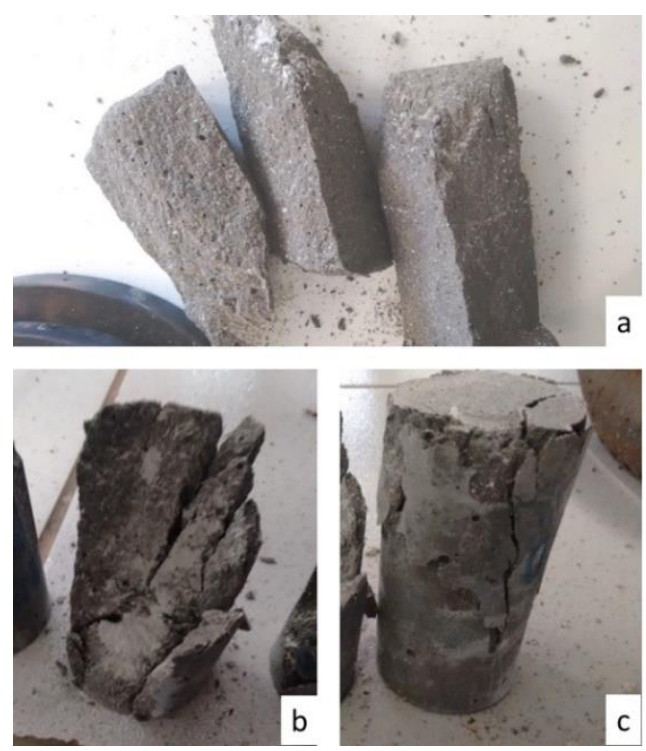

FIGURA 3: Tipologia de ruptura de corpos de prova: (a) sem fibras; (b) 0,5\% de fibras; (c) $1 \%$ de fibras. FONTE: Autoria própria. 
Após a separação manual das faces dos corpos de prova foi possível observar que o aumento na quantidade de fibras resultou em um aumento da quantidade de vazios no concreto (Figura 4). Fato este atribuído ao maior entrelaçamento das mesmas, dificultando o adensamento do material e fazendo com que o concreto, mesmo fluído, não preenchesse completamente o volume dos moldes, influenciando na resistência à compressão dos corpos de prova.

$\mathrm{Na}$ Figura 5 são apresentadas as resistências médias à compressão obtidas e o desvio padrão calculados para cada grupo de concreto, onde se verifica uma pequena variação das resistências entre os diferentes grupos de concreto.

$\mathrm{Na}$ Tabela 4 são apresentados os resultados da análise de variância dos resultados de resistência à compressão dos corpos de prova ensaiados. De acordo com a literatura, quando o valor-P for maior que $5 \%$, não há motivos para rejeitar a hipótese $\mathrm{HO}$, ou seja, não existem diferenças significativas entre os tratamentos. Verifica-se que o valor- $P$ calculado foi de 0,087 $(0,087>0,05)$ e portanto, apesar das variações na resistência à compressão, as resistências dos diferentes grupos de concreto podem ser consideradas iguais.

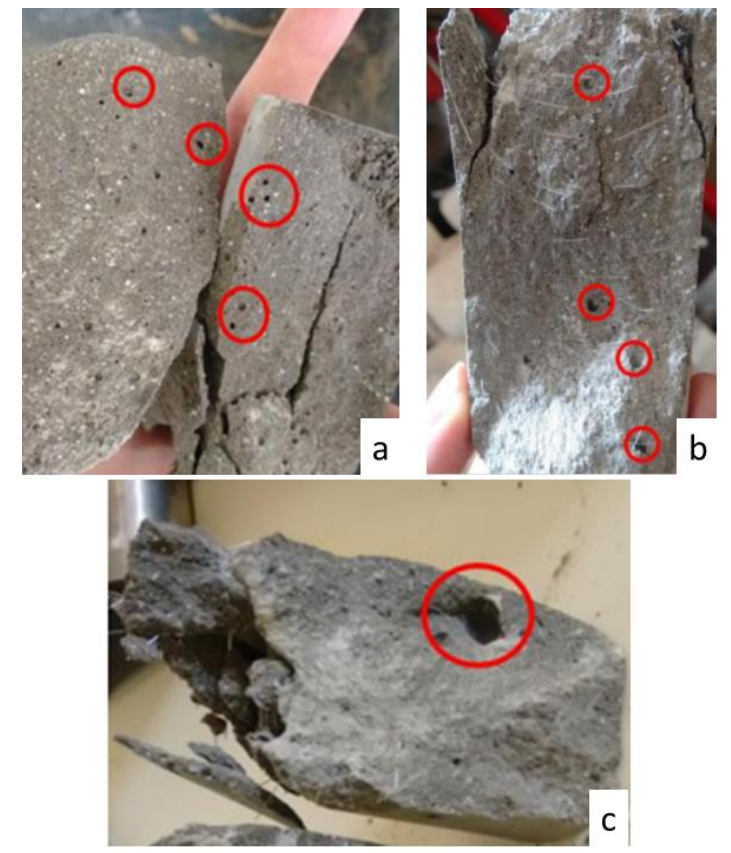

FIGURA 4: Vazios resultantes do entrelaçamento de fibras: (a) Concreto sem fibras; (b) Concreto com 0,5\% de fibras; (c) Concreto com $1 \%$ de fibras.

FONTE: Autoria própria.

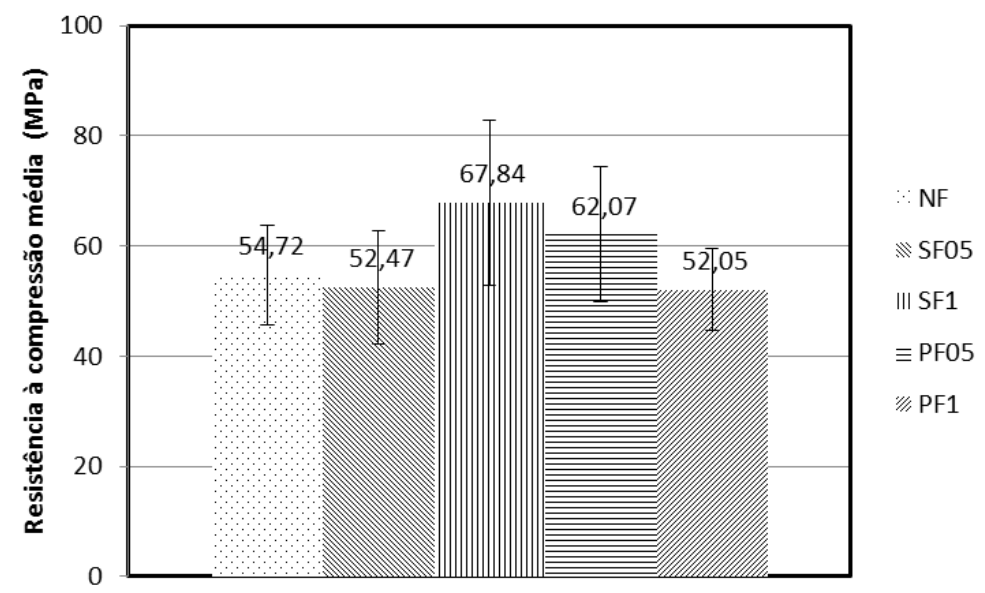

FIGURA 5: Resistência à compressão axial média e desvio padrão dos resultados. FONTE: Autoria própria. 
TABELA 4: Resultado da análise de variância da resistência à compressão.

\begin{tabular}{ccccccc} 
Fonte da variação & $S Q$ & gl & $M Q$ & $F$ & valor-P & F crítico \\
\hline Entre grupos & 1139,948 & 4 & 284,9871 & 2,297227 & 0,087123 & 2,75871 \\
\hline Dentro dos grupos & 3101,424 & 25 & 124,057 & & & \\
\hline Total & 4241,372 & 29 & & &
\end{tabular}

FONTE: Autoria própria.

\subsection{RESISTÊNCIA PÓS-RUPTURA}

As Figuras 6 e 7 apresentam os diagramas de carga versus deslocamento medido durante o ensaio de um dos corpos de prova com adição de $0,5 \%$ e $1 \%$, respectivamente. Observam-se comportamentos distintos após os corpos de prova atingir a máxima resistência no ensaio de compressão. Os corpos de prova não reforçados com fibras esgotaram sua capacidade resistente rompendo bruscamente, sem que haja um nível de resistência residual após a máxima resistência. Isto é perceptível através do grande gradiente decrescente observado no gráfico cargadeslocamento após atingir o máximo carregamento.

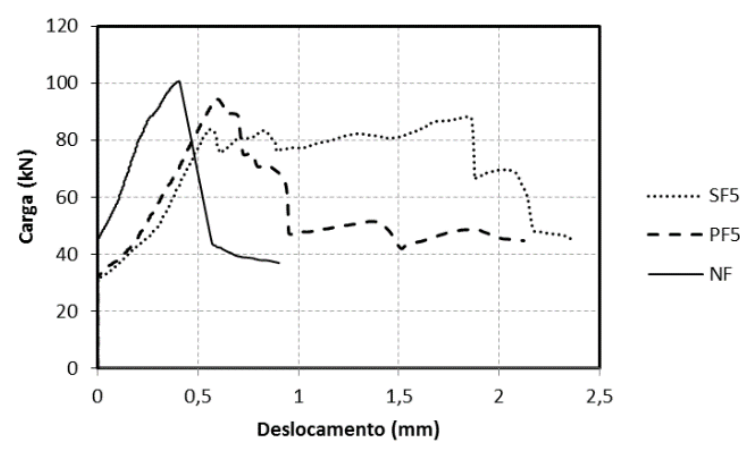

FIGURA 6: Comportamento carga versus deslocamento de corpos de prova sem adição de fibras e com adição de $0,5 \%$ de fibras.

FONTE: Autoria própria.

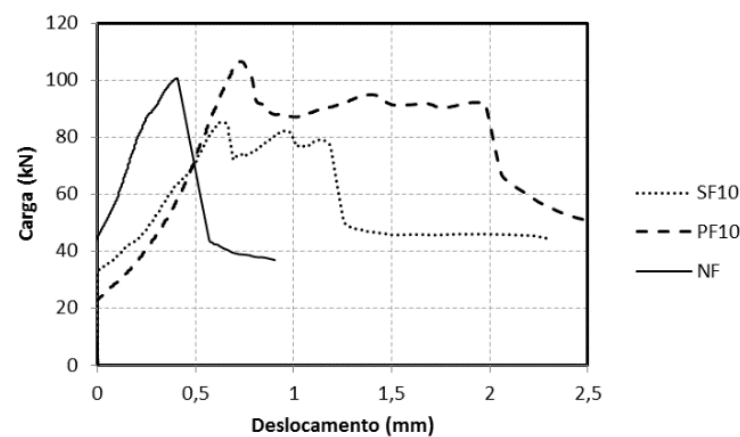

FIGURA 7: Comportamento carga versus deslocamento de corpos de prova sem adição de fibras e com adição de $1 \%$ de fibras.

FONTE: Autoria própria.
Nos concretos reforçados com fibras, independente do material e proporção destas, observa-se que há uma resistência residual após atingir a carga máxima de compressão, da ordem de $90 \mathrm{kN}$, com um comportamento mais dúctil, uma vez que houve maior deformação acompanhada, em algumas ocasiões, de recuperações de resistência passíveis de serem notadas através de picos de carregamento. Tal fato remete ao efeito de aderência das fibras à matriz cimentícia cujo mecanismo demonstra propiciar uma maior ductilidade ao concreto, assim como prover o mesmo de uma resistência residual após esgotar sua capacidade resistente sem que sua ruptura ocorra de forma brusca com separação de suas partes. Além disso, observa-se que tanto as fibras metálicas, quanto poliméricas apresentaram um comportamento próximo no que tange a resistência residual para uma mesma dosagem de fibra, demonstrando que, em ambos os casos o comportamento pós-ruptura é semelhante.

\section{CONCLUSÕES}

Neste trabalho foram analisados os efeitos de fibras de aço e fibras poliméricas sob diferentes dosagens ( $0 \%, 0,5 \%$ e $1 \%$ do volume) na resistência à compressão de concretos de ultra alto desempenho, contribuindo para o desenvolvimento deste material e visando a demanda dos processos construtivos atuais, sendo as principais conclusões apresentadas a seguir:

O concreto de ultra alto desempenho sem adição de fibras apresentou comportamento frágil e passível de rupturas bruscas ao atingir sua máxima resistência. A adoção de fibras, independentemente do material, aumentou a ductilidade do material, evitando separações das partes do concreto após sua ruptura. Observa-se ainda que quanto maior a 
dosagem de fibras utilizada, menor a incidência de separação de partes do concreto.

Devido ao entrelaçamento das fibras foram constatadas dificuldades de adensamento e formação de vazios no interior do concreto, ocorrendo variações na resistência à compressão axial. Assim, a fluidez do concreto no molde tornase uma característica indispensável com o objetivo de reduzir a ocorrência destes vazios.

A adição de fibras não apresentou influência significativa na resistência à compressão axial do concreto em seu regime antes da carga máxima atingida no ensaio, influenciando apenas no comportamento pós-ruptura. Neste ponto, o desempenho de fibras de aço e de polietileno pós ruptura se mostrou semelhante para uma mesma dosagem.

\section{REFERÊNCIAS BIBLIOGRÁFICAS}

Alkaysi, M., El-Tawil S., Liu Z., Hansen W. Effects of silicapowder and cement type on durability of ultra high performance concrete (UHPC). Cement and Concrete Composites 66: 47-56, 2016.

Azad, A. K., Hakeem, I. Y. Flexural Behavior of Hybrid Concrete Beams Reinforced with Ultra-High Performance Concrete Bars. Construction and Building Materials 49: 128-133, 2013.

Bonneau, O., Vernet, C., Moranville, M., Aitcin, P., C. Characterization of the granular packing and percolation threshold of reactive powder concrete. Cement and Concrete Research 30:1861-1867, 2000.

Choi, M. S., Lee, J. S., Ryu, K. S., Koh, K. T., Kwon, S. H. Estimation of rheological properties of UHPC using mini slump test. Construction and Building Materials 106: 632639, 2016.

Dils, J., Boel, V., Schutter, V. Influence of cement type and mixing pressure on air content, rheology and mechanical properties of UHPC. Construction and Building Materials 41: 455-463, 2013.

Habel, K., Viviani, M., Denarié, E., Brühwiler, E. Development of mechanical properties of an Ultra-High Performance Fiber Reinforced Concrete (UHPFRC). Cement and Concrete Research, n. 36, pag. 1362-1370, 2006.

HASSAN, A. M. T., JONES, S. W., MAHMUD, G. H. Experimental Test Methods to Determine the Uniaxial Tensile and Compressive Behaviour of Ultra High Performance Fibre Reinforced Concrete (UHPFRC).
Construction and Building Materials 37: 874-882, 2012.

Kodur, V. K. R., Bhatt, P. P., Soroushian, P., Arablouei, A. Temperature and stress development in ultra-high performance concrete during curing. Construction and Building Materials 122: 63-71, 2016.

Koutný, O., Kratochvíl, J., Svec, J., Bednárek, J. Modelling of packing density for particles composites design. Procedia Engineering 51: 198-205, 2016.

Kusumawardaningsih, Y., Fehling, E., Isamail, M., Aboubakr, A. A. M. Tensile Strength behavior of UHPC and UHPFRC. Procedia Engineering, n. 125, pag. 10811086, 2015.

Larrard, F., Sedran, T. Optimization of ultra-high performance concrete by the use of a packing model. Cement and Concrete Research 24: 997-1009, 1994.

Máca, P., Sovják, R., Vavriník, T. Experimental investigation of Mechanical Properties of UHPFRC. Procedia Engineering, n. 65, pag. 14-19, 2013.

Mehta, P. K., Monteiro, P. J. M. Concrete Microstructure, Properties and Materials. Third Edition. McGraw-Hill, New York, 2006.

Mostofinejad, D., Nikoo, M. R., Hossein, S. A. Determination of optimized mix design and curing conditions of reactive powder concrete (RPC). Construction and Building Materials 123: 754-767, 2016.

Neville, A. M., Brooks, J. J. Concrete Technology. Second Edition, Longman Group, London, 2010.

Ozelame, J. S. Análise experimental da resistência à compressão de concretos de pós-reativos com e sem adição de fibras. Trabalho de conclusão de curso (Graduação em Engenharia Civil) - Universidade Tecnológica Federal do Paraná. Toledo, PR, 2017, 60 p.

Richard, P., Cheyrezi, M. Composition of reactive powder concrete. Cement and Concrete Research 25: 1501-1511, 1995.

Rong, Z., Sun, W., Xiao, H., Jiang, G. Effect of nano-SiO2 particles on the mechanical and microstructural properties of ultra high performance cementitious composites. Cement and Concrete Composites 56: 25-31, 2015.

Schöfl, C., Gruber, M., Plank, J. Preferential adsorption of polycarboxylate superplasticizers on cement and silica fume in ultra-high performance concrete (UHPC). Cement and Concrete Research 42: 1401-1408, 2012.

Soliman, N. A., Tagnit-Hamou, A. Partial substitution of silica fume with fine glass powder in UHPC: filling the micro gap. Construction and Building Materials 139: 374383, 2017.

Su, Y., Li, J., Wu, C., Wu, P., Li, Z. Effect of steel fibres on 
dynamic strength of UHPC. Construction and Building Materials 114: 708-718, 2016.

Tafraoui, A., Escadeillas, G., Vidal, T. Durability of the Ultra High Performance Concrete containing metakaolin. Construction and Building Materials 112: 980-987, 2016.

Wang, D., Shi, C., Wu, Z., Xiao, J., Huang, Z., Fang, Z. A review on ultra high performance concrete: Part II. Hydration, microstructure and properties. Construction and Building Materials 96: 368-377, 2015.

Wang, R., Gao, X., Huang, H., Han, G. Influence of rheological properties of cement mortar on steel fiber distribution in UHPC. Construction and Building Materials 144: 65-73, 2017.

WANG, C., Yang, C., Liu, F., Chaojung, W. Pu, X. Preparation of Ultra High Performance Concrete with Common Technology and Materials. Cement and Concrete Composites, n. 34, pag. 538-544, 2012.

Wille, K., Naaman, A. E., Parra-Montesinos, G. J. Ultra High Performance Concrete With Compressive Strength Exceeding $150 \mathrm{MPa}$ : a Simpler Way. $\mathrm{ACl}$ Materials Journal 108:46-54, 2011.

YANG, I. H., JOH, C., KIM, B. S. Structural Behavior of Ultra High Performance Concrete Beams Subjected to Bending. Engineering Structures, n. 32, pag. 3478-3487, 2010.

Yi, N. H., Kim, J. J., Han, T. S., Lee, J. H. Blast-Resistant Characteristics of Ultra-High Performance Concrete and Reactive Powder Concrete. Construction and Building Materials 28: 694-707, 2012.

Yoo, D., Banthia, N. Mechanical Properties of Ultra-High Performance Fiber Reinforced Concrete: A review. Cement and Concrete Composites, n. 73, pag. 267-280, 2016.

Zbed, T. Ultra High Performance Concrete - Properties and Technology. Bulletin of the Polish Academy of Science. Technical Science 61, n. 1: 183-193, 2013.

Zhou, B., Uchida, Y. Relationship between fiber orientation/distribution and post-cracking behavior in ultra-high performance fiber-reinforced concrete (UHPFRC). Cement and Concrete Composites 83: 66-75, 2017. 